\title{
Usability Design: A New Rational Unified Process Discipline
}

\author{
Magnus Lif ${ }^{1}$ and Bengt Göransson ${ }^{2}$ \\ ${ }^{1}$ Guide Redina, Smedsgränd 9, SE-753 20, Uppsala, Sweden \\ ${ }^{2}$ IT-Arkitekterna, Stora Torget 4, SE-753 20, Uppsala, Sweden \\ Magnus.Lif@guide.se, Bengt.Goransson@it-arkitekterna.se
}

\begin{abstract}
A new discipline, Usability Design, is introduced as an extension to Rational Unified Process (RUP). The aim is to make RUP more user-centred. The discipline springs out of best practices for user-centred design and contains activities that have been carefully tested. Five new roles are introduced to take responsibility for the different activities. An example is given to show the content of the workflow, the workflow details and the activities. The Usability Design discipline help projects to focus on usability and the users throughout the system development lifecycle. In the tutorial the participants will learn how to work with the new discipline within the RUP framework. It will contain practical examples and there will be room for discussions based on the participants own experience.

Use-Centered Systems Design, Usability, Systems Development, Software Engineering, Rational Unified Process
\end{abstract}

\section{Background}

The Rational Unified Process is a software engineering process [3] that significantly has contributed to the software development practice. Today, it is widely used and has contributed to creating a more unified view on software development. However, from a usability point of view the RUP has several drawbacks. Problems with the use of the process have been observed in several projects [2]. It does not provide the support needed to produce a usable system. In a study by Gulliksen, Göransson, Boivie, Blomkvist, Persson \& Cajander [1] the outcome of a project was compared with a list of principles for user-centred design. It shows how a project using RUP with clear intentions to apply a UCD approach ran into several problems that made it difficult to pursue with that approach.

The conclusion from this is that the RUP and use cases as such are not ideal for user-centred design. But, to manage having an impact in practice we have decided to relate our development process to a the RUP based approach, since this is one of the most widely used processes in the large, in-house development organisations we typically work with.

\section{The Usability Design Discipline}

In this tutorial we will introduce a new discipline, Usability Design (for short: UD discipline), extending the RUP. Our aim with the UD discipline is to complement the 
RUP to make it more suitable for systems development where usability is acknowledged to be important. The way to do this is to apply a more user-centred approach to the process. Most of the analysis, design and evaluation work is performed during the inception phase and the early phases of elaboration. During the construction and transition phases there is less work done in the UD discipline. It mainly includes monitoring and making ad hoc design decisions. The actual coding of the GUI is not part of the UD discipline. The proposed discipline is drawn from our previous research and published literature.

\subsection{Roles}

The new roles suggested in the UD discipline are:

- Usability Designer.

- Field Study Specialist.

- Interaction Designer.

- Graphic Designer.

- Usability Evaluation Specialist.

\subsection{The Workflow}

The workflow includes the user-centred activites performed in each iteration of the UD discipline.

The activities are:

- Usability design plan.

- Conduct user studies.

- Perform competitor analysis.

- Conceptual design.

- Interaction design.

- Detailed design.

- Develop user assistance.

- Monitor usability work.

- Usability evaluation.

\section{References}

[1] Gulliksen, J., Göransson, B., Boivie, I., Blomkvist, S., Persson, J., Cajander, Å.: Key Principles For User Centred Systems Design. In: Special Section Designing It For Healthy Work, In Behaviour \& Information Technology, November-December 2003, vol. 22(6), pp. 397-409, Taylor \& Francis (2003)

[2] Gulliksen, J., Göransson, B., Lif, M.: A User-Centered Approach To Object-Oriented User Interface Design. In: Van Harmelen, M. (ed.) Designing Interactive Systems: Object Modeling And User Interface Design, Addison-Wesley, Reading (2001)

[3] Kruchten, P.: The Rational Unified Process-An Introduction. Addison Wesley Longman Inc., Reading, Mass., Usa (1998) 